\title{
Uma proposta alternativa para a classificação de cônicas por meio de números complexos
}

\author{
Tacildo de Souza Araújo ${ }^{1}$ \\ IMECC/UNICAMP, Campinas, SP \\ Cristiano Torezzan ${ }^{2}$ \\ FCA/UNICAMP, Limeira, SP
}

\begin{abstract}
Resumo. Neste trabalho apresentamos uma conexão entre números complexos e cônicas. De forma didática e sem abandonar completamente o formalismo matemático, utilizaremos a estrutura geométrica dos números complexos para construir uma sequência de resultados que fornecem uma alternativa elegante e acessível para caracterizar e classificar as cônicas. Nosso método permite a compreensão e realização de movimentos de rotação e translação das cônicas por meio de operações elementares com números complexos, ressaltando assim, o poder de tais números na resolução de problemas envolvendo movimentos no plano. Espera-se que este trabalho sirva como ferramenta alternativa para o ensino-aprendizado de cônicas e números complexos, contribuindo para a valorização da criatividade de estudantes e professores na elaboração de ferramentas alternativas que enriqueçam o ensino da matemática.
\end{abstract}

Palavras-chave. Ensino de matemática, Números Complexos, Cônicas.

\section{Introdução}

Os primeiros estudos sobre cônicas remontam aos trabalhos de Apolônio de Perga (261 aC), cujo resultado compilado ficou conhecido na forma do Tratado sobre Cônicas, [3]. Séculos mais tarde, o estudo analítico das cônicas foi apresentado por Fermat (1601-1665), que determinou expressões gerais para a hipérbole, elipse e parábola por meio de equações do segundo grau nas coordenadas $(x, y)$ do plano [3].

De maneira geral, uma cônica é o lugar geométrico dos pontos $(x, y)$ do plano real, que satisfazem a equação $a x^{2}+b x y+c y^{2}+d x+e y+f=0$, com $a, b, c, d, e, f \in \mathbb{R}$, não simultaneamente nulos. Geometricamente, as cônicas são obtidas pela interseção de um plano com um cone duplo de revolução, originando três diferentes curvas: elipses, parábolas e hipérboles.

Para além de sua intrínseca beleza geométrica e ampla aplicabilidade, do ponto de vista analítico, um dos problemas fundamentais no estudo de cônicas refere-se à caracterização e classificação dessas figuras com base em sua representação algébrica. De maneira geral, dada uma equação completa de segundo grau em duas variáveis, deseja-se determinar qual a cônica associada e caracterizar as propriedades da curva subjacente.

O problema de classificação de cônicas aparece pela primeira no Ensino Médio, de forma quase concomitante com a introdução dos números complexos. Nesse contexto, uma conexão entre esses dois temas pode ser muito oportuna para explorar os aspectos geométricos dos números complexos e apresentar uma forma alternativa para a caracterização de cônicas e determinação dos seus pontos

\footnotetext{
${ }^{1}$ tacildo.araujo@ifam.edu.br

2 torezzan@unicamp.br
} 
notáveis sem utilizar a abordagem clássica através autovalores. É esse o propósito do presente trabalho.

Na seção 2 estabelecemos as notações e apresentamos alguns resultados preliminares que formam a base para o desenvolvimento do nosso método. A seção 3 traz as principais contribuições do trabalho. Nessa seção discutimos de forma breve a caracterização, a classificação e as condições de existência das cônicas e exploramos os casos degenerados mais importantes. Nas seções 4 e 5 apresentamos um roteiro, seguido de um exemplo, para ilustração do método e as conclusões do trabalho.

\section{Preliminares}

Nesta seção apresentamos os fundamentos matemáticos que formam a base para o método que será desenvolvido ao longo do trabalho. Para um estudo mais detalhado e aprofundado recomendamos $[1,2,4]$.

Denotaremos por $\mathbb{R}$ e $\mathbb{C}$ o conjunto dos números reais e o conjunto dos números complexos, respectivamente. Dado $z=a+b i \in \mathbb{C}, i$ é chamado de unidade imaginária, o conjugado de $z$ será denotado por $\bar{z}=a-b i, a=\operatorname{Re}(z)$ e $b=\operatorname{Im}(z)$ são, respectivamente, as partes real e imaginária de $z$.

É possível verificar, por substituição direta, que $a=\frac{z+\bar{z}}{2}$ e $b=\frac{z-\bar{z}}{2 i}$. Estas fórmulas serão especialmente úteis ao longo deste trabalho.

Como primeira contribuição deste trabalho apresentamos uma fórmula para o cálculo da raiz de um número complexo que será muito utilizada na seção 4.

Proposição 2.1. Dado $z=a+b i \in \mathbb{C}$ não nulo. Uma das raízes de z é dada por

$$
\sqrt{a+b i}=\sqrt{\frac{\sqrt{a^{2}+b^{2}}+a}{2}}+\operatorname{sig}(b) \sqrt{\frac{\sqrt{a^{2}+b^{2}}-a}{2}} i
$$

onde sig(b) é o sinal da parte imaginária b do complexo z.

As próximas duas proposições constituem os principais pilares do trabalho. Por um lado, a Proposição 2.2 fornece um fórmula para a distância de uma reta a um ponto arbitrário fora dela por meio de números complexos. Por outro lado, a Proposição 2.3 oferece uma definição menos conhecida, porém, mais geral para o conceito de cônica. De fato, a proposição mostra que elipse, hipérbole e parábola fazem parte da mesma família, como mostra a figura 1.

Proposição 2.2. Seja $r$ uma reta em $\mathbb{C}$ definida por $z(t)=z_{0}+t v$ com $|v|=1$ e $t \in \mathbb{R}$. A distância mínima $\lambda$ de um ponto arbitrário $f$ à reta $r$ é dada pela equação

$$
\lambda=\left|\operatorname{Im}\left(\left(f-z_{0}\right) \bar{v}\right)\right|
$$

Proposição 2.3. Sejam $r$ uma reta do plano complexo, $f$ um ponto fora dela e $\varepsilon>0$ um número real. Se $z \neq f$ é um ponto arbitrário fora de $r$, então a equação $\frac{d(z, f)}{d(z, r)}=\varepsilon$, possui solução. Além disso, a solução é uma elipse para $0<\epsilon<1$, uma parábola para $\epsilon=1$ e uma hipérbole para $\epsilon>1$.

Em função da limitação de páginas deste trabalho, omitiremos a demonstração das proposições acima, que podem ser encontradas em [1]. 


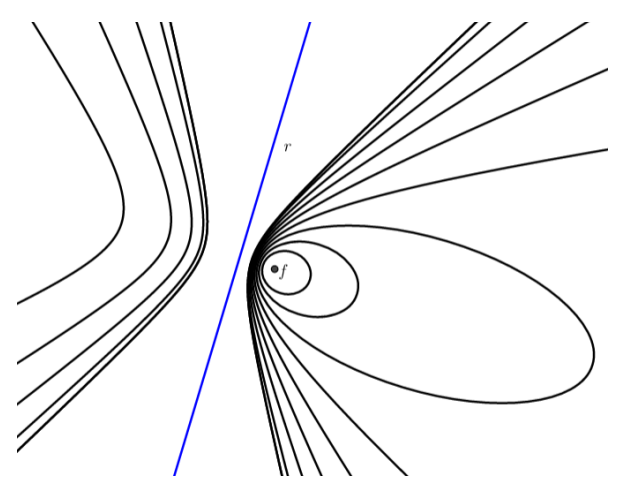

Figura 1: Família de cônicas

\section{Números complexos e cônicas}

Nesta seção desenvolveremos os conceitos e resultados que permitem analisar a existência, caracterizar e classificar as cônicas através de uma equação envolvendo termos quadráticos e lineares da variável $z \in \mathbb{C}$.

\subsection{A forma padrão de uma cônica}

Considere a equação dada por

$$
\bar{A} z^{2}+A \bar{z}^{2}+B z \bar{z}+C z+\bar{C} \bar{z}+D=0,
$$

com $A, C \in \mathbb{C}$ e $B, D \in \mathbb{R}$. Mostraremos que, se $C$ é uma cônica, então sua equação satisfaz a equação (2). Com efeito, sejam $r$ a reta diretriz da cônica, definida por $r(t)=z_{0}+t v$, com $z_{0}, v \in \mathbb{C},|v|=1$ e $f$ um ponto (foco) fora dela. Usando a Proposição 2.3 e em seguida a Proposição 2.2 , obtemos a equação

$$
|z-f|=\varepsilon\left|\operatorname{Im}\left(\left(z-z_{0}\right) \bar{v}\right)\right| .
$$

Elevando ao quadrado ambos os lados da equação (3), usando $|z|^{2}=z \bar{z}$ e $\operatorname{Im}\left(\left(z-z_{0}\right) \bar{v}\right)=$ $\frac{\left(z-z_{0}\right) \bar{v}-\left(\bar{z}-\overline{z_{0}}\right) v}{2 i}$, após algumas simplificações obtemos

$$
\begin{aligned}
& \bar{v}^{2} z^{2}+v^{2} \bar{z}^{2}+\left(\frac{4}{\varepsilon^{2}}-2\right) z \bar{z}+\left(2 \overline{z_{0}}-2 z_{0} \bar{v}^{2}-\frac{4}{\varepsilon^{2}} \bar{f}\right) z+\left(2 z_{0}-2 \overline{z_{0}} v^{2}-\frac{4}{\varepsilon^{2}} f\right) \bar{z}+ \\
& \left(\frac{4}{\varepsilon^{2}} f \bar{f}+z_{0}^{2} \bar{v}^{2}+\bar{z}_{0}^{2} v^{2}-2 z_{0} \overline{z_{0}}\right)=0 .
\end{aligned}
$$

Tomando $A=v^{2}, B=\frac{4}{\varepsilon^{2}}-2>0, C=2 \overline{z_{0}}-2 z_{0} \bar{v}^{2}-\frac{4}{\varepsilon^{2}} \bar{f}$ e $D=\frac{4}{\varepsilon^{2}} f \bar{f}+z_{0}^{2} \bar{v}^{2}+\bar{z}_{0}^{2} v^{2}-2 z_{0} \overline{z_{0}}$, temos o resultado desejado.

A recíproca do resultado acima não vale, isto é, nem sempre a equação (2) representa uma cônica. Quando for o caso, a classificação da cônica se dá facilmente pela observação do valor de $B$. Com efeito, pela Proposição 2.3, $\epsilon=1$ implica $B=2$ (parábola), $0<\epsilon<1$ implica $B>2$ (elipse) e $\epsilon>1$ implica $-2<B<2$ (hipérbole).

Diremos que a equação (2) está na forma padrão quando $|A|=1$ e $B>-2$. Note que se $A \neq 0$ e $B \leq-2$, então a equação $-|A|^{-1}\left(\bar{A} z^{2}+A \bar{z}^{2}+B z \bar{z}+C z+\bar{C} \bar{z}+D\right)=0$, está na forma padrão. Portanto, toda equação da forma (2) pode ser escrita na forma padrão. Mais ainda, se $A \neq 0$ a equação (2) não representa uma cônica. Um estudo detalhado dos casos degenerados decorrentes desta situação pode ser encontrado em [1]. 
De posse desses resultados, podemos fazer a conexão com o estudo clássico das cônicas. Considere a equação geral do segundo grau com duas variáveis com coeficientes reais dada por

$$
a x^{2}+b x y+c y^{2}+d x+e y+f=0 .
$$

Substituindo $x=\frac{z+\bar{z}}{2}$ e $y=\frac{z-\bar{z}}{2 i}$ na equação (4) e reagrupando os termos semelhantes, obtemos

$$
\left(\frac{a-c-b i}{4}\right) z^{2}+\left(\frac{a-c+b i}{4}\right) \bar{z}^{2}+\left(\frac{2 a+2 c}{4}\right) z \bar{z}+\left(\frac{d-e i}{2}\right) z+\left(\frac{d+e i}{2}\right) \bar{z}+f=0 .
$$

Comparando as equações (5) e (2) obtém-se $A=\frac{a-c+b i}{4}$. Portanto, de acordo com o que vimos acima, uma condição necessária para que a equação (4) represente uma cônica é $(a-c)^{2}+b^{2} \neq 0$.

\subsection{A forma canônica de uma cônica}

Suponha que a equação (2) esteja na forma padrão. Fazendo a mudança de variável $z=$ $\sqrt{A} w \Rightarrow z^{2}=A w^{2}$ e observando que $\bar{z}^{2}=\bar{A} \bar{w}^{2}$ e $\bar{z}=\overline{\sqrt{A}} \bar{w}=\sqrt{\bar{A}} \bar{w}$, temos

$$
\bar{A} A w^{2}+A \bar{A} \bar{w}^{2}+B \sqrt{A} w \overline{\sqrt{A}} \bar{w}+C \sqrt{A} w+\bar{C} \overline{\sqrt{A}} \bar{w}+D=0
$$

Note que $\sqrt{A} \sqrt{\bar{A}}=\sqrt{A \bar{A}}=\sqrt{|A|^{2}}=|A|=1$. Assim, tomando $C^{\prime}=C \sqrt{A}$ obtém-se a equação

$$
w^{2}+\bar{w}^{2}+B w \bar{w}+C^{\prime} w+\overline{C^{\prime}} \bar{w}+D=0
$$

, que chamaremos de forma canônica.

Substituindo $w=x_{1}+y_{1} i$ e $C^{\prime}=c_{1}^{\prime}+c_{2}^{\prime} i$ na equação acima obtemos

$$
(B+2) x_{1}^{2}+(B-2) y_{1}^{2}+2 c_{1}^{\prime} x_{1}-2 c_{2}^{\prime} y_{1}+D=0 .
$$

Fazendo $\lambda_{1}=B+2>0$ e $\lambda_{2}=B-2>-4$ a equação resulta em

$$
\lambda_{1} x_{1}^{2}+\lambda_{2} y_{1}^{2}+2 c_{1}^{\prime} x_{1}-2 c_{2}^{\prime} y_{1}+D=0 .
$$

Resta analisar a equação (7) para os diferentes valores de $B$. Se $B>2\left(\lambda_{2}>0\right)$ a equação se reduz a

$$
\lambda_{1} x_{2}^{2}+\lambda_{2} y_{2}^{2}=D^{\prime}
$$

com $D^{\prime}=\frac{c_{1}^{\prime 2}}{\lambda_{1}}+\frac{c_{2}^{\prime 2}}{\lambda_{2}}-D$ e $u=x_{2}+y_{2} i$ é dado pela translação $u=w+\left(\frac{c_{1}^{\prime}}{\lambda_{1}}-\frac{c_{2}^{\prime}}{\lambda_{2}} i\right)$. Portanto, se $D^{\prime}<0$ a equação não tem solução, se $D^{\prime}=0$ a equação possui solução única $x_{2}=y_{2}=0$ e se $D^{\prime}>0$ obtemos a elipse de equação

$$
\frac{x_{2}^{2}}{\left(\sqrt{\frac{D^{\prime}}{\lambda_{1}}}\right)^{2}}+\frac{y_{2}^{2}}{\left(\sqrt{\frac{D^{\prime}}{\lambda_{2}}}\right)^{2}}=1 .
$$

Por outro lado, se $-2<B<2\left(\lambda_{1}>0\right.$ e $\left.\lambda_{2}<0\right)$, então a equação (7) se reduz a

$$
\lambda_{1} x_{2}^{2}-\left|\lambda_{2}\right| y_{2}^{2}=D^{\prime} .
$$


Logo, $D^{\prime}=0$, implica $y_{2}= \pm \sqrt{\frac{\lambda_{1}}{\left|\lambda_{2}\right|}} x$, ou seja, a equação representa um par de retas. Por outro lado, se $D^{\prime} \neq 0$, então dividimos a equação (9) por $D^{\prime}$ e obtemos a hipérbole de equação

$$
\frac{x_{2}^{2}}{\left(\sqrt{\frac{D^{\prime}}{\lambda_{1}}}\right)^{2}}-\frac{y_{2}^{2}}{\left(\sqrt{\frac{D^{\prime}}{\left|\lambda_{2}\right|}}\right)^{2}}=1 .
$$

Finalmente, se $B=2\left(\lambda_{2}=0\right)$ após o completamento de quadrados, a equação (7) tem forma

$$
\lambda_{1}\left(x_{1}+\frac{c_{1}^{\prime}}{\lambda_{1}}\right)^{2}-2 c_{2}^{\prime} y_{1}+D-\frac{c_{2}^{\prime 2}}{\lambda_{1}}=0
$$

daí, se $c_{2}^{\prime}=0$ a equação não representa uma cônica e se $c_{2}^{\prime} \neq 0$, dividimos a equação por $2 c_{2}^{\prime}$ e obtemos a parábola de equação

$$
x_{2}^{2}=2 p y_{2},
$$

$\operatorname{com} 2 p=\frac{2 c_{2}^{\prime}}{\lambda_{1}}$ e $u=w+\left(\frac{c_{1}^{\prime}}{\lambda_{1}}-\left(\frac{\lambda_{1} D-c_{2}^{\prime 2}}{2 \lambda_{1} c_{2}^{\prime}}\right) i\right)=x_{2}+y_{2} i$.

Para facilitar as aplicações, resumimos os resultados desta seção na tabela abaixo. Para isso, considere $B>-2$ e $C^{\prime}=c_{1}^{\prime}+c_{2}^{\prime} i$ na equação (6).

Tabela 1: Classificação das cônicas

\begin{tabular}{ccc}
\hline$B$ & Cônica & Se e somente se \\
\hline$-2<B<2$ & Elipse & $\frac{{c_{1}^{\prime}}^{2}}{B+2}+\frac{{c_{2}^{\prime}}^{2}}{B-2}-D \neq 0$ \\
$B=2$ & Parábola & $c_{2}^{\prime}=\operatorname{Im}\left(C^{\prime}\right) \neq 0$ \\
$B>2$ & Hipérbole & $\frac{{\frac{c_{1}^{\prime}}{}}^{2}}{B+2}+\frac{{c_{2}^{\prime}}^{2}}{B-2}-D>0$ \\
\hline
\end{tabular}

\section{Roteiro para classificação de cônicas por meio de números complexos}

Nesta seção apresentamos um processo de caracterização, classificação e determinação dos pontos notáveis das cônicas através de números complexos. Além disso, apresentamos um exemplo para ilustrar o método proposto.

\subsection{Roteiro}

- Passo 1: verificar se $(a-c)^{2}+b^{2}$ é diferente de zero.

- Passo 2: Complexificar a equação tomando $x=\frac{z+\bar{z}}{2}$ e $y=\frac{z-\bar{z}}{2 i}$.

- Passo 3: Colocar a equação na forma padrão (Dividindo a equação pelo módulo de $A$ e multiplicando por -1 se $B \leq-2$ ). 
- Passo 4: Aplicar a rotação $z=\sqrt{A} w$ para obter a forma canônica.

- Passo 5: Verificar na tabela 1 se a equação na forma canônica representa uma cônica.

- Passo 6: Transformar a forma canônica em coordenadas cartesianas e completar os quadrados.

- Passo 7: Aplicar a translação $u=w+\gamma$ para obter a equação da cônica no plano $U$.

- Passo 8: Determinar todos os elementos na cônica nesse último sistema de coordenadas.

- Passo 9: Aplicar a isometria inversa para obter os elementos no sistema de coordenadas original.

\subsection{Exemplo}

Dada a equação $2 x^{2}+2 y^{2}-4 x y+8 y=0$, vamos investigar se há uma cônica associada. Em caso afirmativo, quais são as suas características. Seguiremos o roteiro apresentado previamente.

Passo 1: Como $(a-c)^{2}+b^{2}=(2-2)^{2}+4^{2}=16$, a equação pode representar uma cônica.

Passo 2: Complexificando a equação obtemos,

$$
4 i z^{2}+(-4 i) \bar{z}^{2}+8 z \bar{z}+(-16 i) z+(16 i) \bar{z}=0
$$

Passo 3: Dividindo-se a equação acima por $|A|=|-4 i|=4$, obtém-se a forma padrão,

$$
i z^{2}+(-i) \bar{z}^{2}+2 z \bar{z}+(-4 i) z+(4 i) \bar{z}=0 .
$$

Pela equação (1), a direção da diretriz é dada por $v=\sqrt{A}=\sqrt{-i}=\frac{1}{\sqrt{2}}-\frac{1}{\sqrt{2}} i$.

Passo 4: Fazendo $z=\sqrt{A} w$, obtemos a forma canônica da cônica

$$
w^{2}+\bar{w}^{2}+2 w \bar{w}+\left(-\frac{4}{\sqrt{2}}-\frac{4}{\sqrt{2}} i\right) w+\left(-\frac{4}{\sqrt{2}}+\frac{4}{\sqrt{2}} i\right) \bar{w}=0
$$

Passo 5: Note que $B=2$ e $c_{2}^{\prime}=-4 / \sqrt{2}$ implicam que a cônica é uma parábola, conforme Tabela 1).

Passo 6: Fazendo $w=x_{1}+y_{1} i$ e completando o quadrado obtém-se a equação da parábola, no plano $W$, dada por $\left(x_{1}-\frac{\sqrt{2}}{2}\right)^{2}=-\sqrt{2}\left(y_{1}-\frac{\sqrt{2}}{4}\right)$.

Passo 7: Aplicando a translação

$$
u=w+\left(-\frac{\sqrt{2}}{2}-\frac{\sqrt{2}}{4} i\right)=\left(x^{\prime}-\frac{\sqrt{2}}{2}\right)+\left(y^{\prime}-\frac{\sqrt{2}}{4}\right) i=x_{2}+y_{2} i,
$$

obtemos a equação da parábola no plano $U$,

$$
x_{2}^{2}=-\sqrt{2} y_{2} .
$$

Passo 8: A equação (12) permite a determinação de todos os pontos notáveis da parábola no plano $U$.

Passo 9: Em seguida, usa-se a equação (11) para determinar tais pontos no plano $W$ e, finalmente, usa-se $z=\sqrt{A} w$ para obter os pontos no plano original (Plano complexo)

Evidentemente, os pontos no plano original podem ser determinados por meio da composição de movimentos dada por $z=\left(\frac{1}{\sqrt{2}}-\frac{1}{\sqrt{2}} i\right)\left[u+\left(\frac{\sqrt{2}}{2}+\frac{\sqrt{2}}{4} i\right)\right]$. Entretanto, a sequência de passos 



Figura 2: Parábola nos planos $U, W$ e complexo, respectivamente.

recomendada permite que o aluno perceba os movimentos (rotação e translação) que estão sendo realizados ao longo do processo de obtenção dos pontos. A figura abaixo ilustra esses movimentos.

A figura (b) representa a translação do plano $U$ (figura (a)) da direção do complexo $\gamma=$ $\frac{\sqrt{2}}{2}+\frac{\sqrt{2}}{4} i$ e a figura (c) representa a rotação do plano $W$ (figura (b)) de um ângulo de 45 graus, no sentido horário.

\section{Conclusões}

Propomos um método alternativo, acessível e eficiente para caracterizar e classificar as cônicas por meio de operações elementares com números complexos. Elaboramos um passo a passo que permite que o aluno construa o conhecimento sem a necessidade de decorar fórmulas, além de valorizar o aspecto geométrico dos números complexos, tão pouco explorado no ensino.

\section{Agradecimentos}

Agradecemos ao professor Alfredo Wagner Martins Pinto (in memorian) pelas contribuições em parte deste trabalho.

\section{Referências}

[1] Araújo, Tacildo de Souza. Números complexos e cônicas. Dissertação de Mestrado. UFAM, 2014.

[2] Andreescu, Titu, Andrica, Dorin. Complex Number from A to... Z, volume 165. Boston, Birkhäuser, 2006.

[3] Boyer, Carl B. História da Matemática, Edgar Bluncher Ltda, São Paulo, 1996.

[4] Yaglom, Isaak Moiseevitch.Complex numbers in geometry. Academic Press, 2014. 